\title{
EXPANDING SOLITONS WITH NON-NEGATIVE CURVATURE OPERATOR COMING OUT OF CONES
}

\author{
FELIX SCHULZE AND MILES SIMON
}

\begin{abstract}
We consider Ricci flow of complete Riemannian manifolds which have bounded non-negative curvature operator, non-zero asymptotic volume ratio and no boundary. We prove scale invariant estimates for these solutions. Using these estimates, we show that there is a limit solution, obtained by scaling down this solution at a fixed point in space. This limit solution is an expanding soliton coming out of the asymptotic cone at infinity.
\end{abstract}

\section{INTRODUCTION AND STATEMENT OF RESULTS}

Let $\left(M^{n}, h\right)$ be a smooth $n$-dimensional, complete, non-compact Riemannian manifold without boundary, with non-negative curvature operator and bounded curvature. In particular $(M, h)$ has non-negative sectional curvature and non-negative Ricci curvature. Any rescaling of this space also has non-negative sectional curvatures, and hence for every sequence of scalings $\left(M, c_{i} h, p_{i}\right), c_{i} \in \mathbb{R}^{+}, p_{i} \in M, i \in \mathbb{N}$, there exists a subsequence which converges in the pointed Gromov Hausdorff sense to a metric space $\left(X, d_{X}\right)$ (see Proposition 10.7.1 in [2]) which is a metric space with curvature $\geq 0$ (see Definition 4.6 .2 in $[2]$ ). In the case that $c_{i} \rightarrow 0$ and $p_{i}=p$ for all $i \in \mathbb{N}$, the limit is known as $\left(X, d_{X}, 0\right)$ the asymptotic cone at infinity and it is unique: see Lemma 3.4 of [17]. It is the Euclidean cone over a metric space $\left(V, d_{V}\right)$ where $\left(V, d_{V}\right)$ is an Alexandrov space of curvature bounded from below by one, and 0 is the tip of the cone: see Corollary 3.5 of [17] for example.

The Euclidean cone $C V$ over a metric space $V$ is homeomorphic to the space $\mathbb{R}_{0}^{+} \times V / \sim$ with the quotient topology, where $(r, y) \sim(s, x)$ if and only if $(r=s=0)$ or $(r=s$ and $x=y)$. The metric is given by:

$$
d_{C V}((r, x),(s, y)):=r^{2}+s^{2}-2 r s \cos \left(\min \left(d_{V}(x, y), \pi\right)\right) .
$$

In the case that $C V$ arises as a Gromov Hausdorff limit in the setting described above, and the sequence $\left(M, c_{i} h, p_{i}\right)$ is non-collapsing, then $V$ is homeomorphic to $S^{n-1}$. This follows from (unpublished) results of G. Perelman [27] as explained and simplified by V. Kapovitch in the paper 22. See Appendix B]

Notation: A pointed sequence $\left(X_{i}, d_{i}, p_{i}\right)$ of metric spaces is non-collapsing if $\operatorname{vol}\left(B_{1}(p)\right) \geq \delta>0$ for all $p \in X_{i}$ and some $\delta>0$ independent of $i$.

Our aim is to flow such cones $\left(C V, d_{C V}\right)$ by Hamilton's Ricci flow (introduced in [18]). We will show that a solution to Ricci flow with initial value given by the cone exists, that the solution is immediately smooth, and that it is an expanding

2000 Mathematics Subject Classification. 53C44, 35Bxx.

Key words and phrases. Ricci flow, expanding solitons, asymptotic cone. 
Ricci soliton with non-negative curvature operator. For an interval $I$ (open, closed, half open, finite or infinite length) and a smooth manifold $M$ without boundary, a smooth (in space and time) family of complete Riemannian metrics $\{g(\cdot, t)\}_{t \in I}$ solves Ricci flow if $\frac{\partial}{\partial t} g(t)=-2 \operatorname{Ric}(\mathrm{g}(\mathrm{t}))$ for all $t \in I$. If $I=(0, T)$, we say $(M, g(t))_{t \in(0, T)}$ is a solution to Ricci flow with initial value $\left(M, d_{0}\right)\left(\left(M, d_{0}\right)\right.$ a metric space), if $\lim _{t \searrow 0}(M, d(g(t)))=\left(M, d_{0}\right)$ in the Gromov-Hausdorff sense $((M, d(g))$ is the metric space associated to $(M, g))$.

Definition 1.1. Let $(M, h)$ be a smooth, complete Riemannian manifold without boundary, with non-negative Ricci curvature. The asymptotic volume ratio $\operatorname{AVR}(M, h)$ is

$$
\operatorname{AVR}(M, h):=\lim _{r \rightarrow \infty} \frac{\operatorname{vol}\left(B_{r}(x)\right)}{r^{n}},
$$

where $x$ is an arbitrary point in $M$.

Due to the Bishop Gromov volume comparison principle, $\operatorname{AVR}(M, h)$ is well defined for such manifolds, and does not depend on the point $x$. It also easily follows that

$$
\frac{\operatorname{vol}\left(B_{r}(x)\right)}{r^{n}} \geq \operatorname{AVR}(M, h) \quad \forall r>0 .
$$

In particular if $V_{0}:=\operatorname{AVR}(M, h)>0$, then we have

$$
\frac{\operatorname{vol}\left(B_{r}(x)\right)}{r^{n}} \geq V_{0}>0 \quad \forall r>0 .
$$

Theorem 1.2. Let $(M, h)$ be a smooth, complete Riemannian manifold without boundary, with non-negative, bounded curvature operator and positive asymptotic volume ratio $V_{0}:=\operatorname{AVR}(M, h)>0$. Let $\left(X, d_{X}, 0\right)$ be the asymptotic cone at infinity, i.e. the unique Gromov-Hausdorff limit of $\left(M, c_{i} h, p_{0}\right)$ for any sequence $c_{i} \rightarrow 0$ of positive numbers and any base point $p_{0} \in M$. Then:

(i) There exists a smooth solution $(M, g(t))_{t \in[0, \infty)}$ to Ricci flow with $g(0)=h$.

(ii) Let $^{i}(t):=c_{i} g\left(t / c_{i}\right), i \in \mathbb{N}$, be the solutions to Ricci flow obtained by rescaling the flows obtained in (i). The pointed solutions $\left(M, g_{i}(t), p_{0}\right)_{t \in(0, \infty)}$ converge smoothly, sub-sequentially (in the Hamilton-Cheeger-Gromov sense: see [20]) as $i \rightarrow \infty$ to a limit solution $\left(\tilde{X}, \tilde{g}(t)_{t \in(0, \infty)}, \tilde{x}_{0}\right)$. This solution $\left(\tilde{X}, \tilde{g}(t)_{t \in(0, \infty)}, \tilde{x}_{0}\right)$ satisfies $\left(\tilde{X}, d(\tilde{g}(t)), \tilde{x}_{0}\right) \rightarrow\left(X, d_{X}, 0\right)$ in the GromovHausdorff sense as $t \searrow 0, A V R(\tilde{X}, \tilde{g}(t))=A V R\left(X, d_{X}\right)=A V R(M, h) \forall t>$ 0 , and $X$ is homeomorphic to $\tilde{X}$. Hence such a limit solution may be thought of as a solution to Ricci flow with initial value $\left(X, d_{X}, 0\right)$. Furthermore, $(\tilde{X}, \tilde{g}(t))_{t \in(0, \infty)}$ is an expanding gradient soliton with non-negative curvature operator. That is $\tilde{g}(t)=t\left(\phi_{t}\right)^{*} \tilde{g}(1)$ and $\tilde{g}(1)$ satisfies

$$
\operatorname{Ric}(\tilde{\mathrm{g}}(1))-(1 / 2) \tilde{\mathrm{g}}(1)+{ }^{\tilde{\mathrm{g}}(1)} \nabla^{2} \mathrm{f}
$$

for some smooth function $f: M \rightarrow \mathbb{R}$.

Remark 1.3. As explained before, in this case $X$ is the Euclidean cone over a metric space $V$, and $V$ is homeomorphic to $S^{n-1}$, see Appendix B. Thus $X$ is homeomorphic to $\mathbb{R}^{n}$.

In this paper (and in particular the above theorem) ${ }^{g} \nabla^{j}$ refers to the $j$ th covariant derivative with respect to $g$. In order to prove this theorem we require a priori estimates for non-collapsed solutions with non-negative bounded curvature operator. 
This involves proving a refined version of Lemma 4.3 of [30, suited to the current setting, which we now state.

Theorem 1.4. Let $\left(M^{n}, g_{0}\right)$ be a smooth, complete Riemannian manifold without boundary, with bounded non-negative curvature operator and $V_{0}:=\operatorname{AVR}\left(M, g_{0}\right)>$ 0 . Then there exists a constant $c=c\left(n, V_{0}\right)>0$ and a solution to Ricci-flow $(M, g(t))_{t \in[0, \infty)}$ with $g(0)=g_{0}$ such that

$$
\sup _{M}|\operatorname{Riem}(g(t))| \leq \frac{c}{t},
$$

for all $t \in(0, \infty)$.

It is known that any smooth, open solution to Ricci flow with non-negative, bounded curvature operator has constant asymptotic volume ratio, see [32, Theorem 7]. That is, in the above theorem we have

$$
V_{t}:=\operatorname{AVR}(M, g(t))=V_{0},
$$

for all $t \in[0, \infty)$.

After completing this paper a pre-print of E. Cabezas-Rivas and B. Wilking appeared [3] where the necessary a priori estimates are shown to extend our results to open Riemannian manifolds with non-negative (possibly unbounded) complex sectional curvature .

\section{Previous Results and structure of the Paper}

The literature that exists on expanding, shrinking and steady solitons is vast. For a very good and current overview of the field, we refer the reader to the survey paper of H-D. Cao [6]. Here we mention some of the results on expanding solitons relevant to the current setting.

In the paper [4, the author constructs families of examples of Kähler gradient expanding solitons on $\mathbb{C}^{n}$. He also shows that any solution to Kähler Ricci flow with bounded curvature and which

(i) exists for $t \in(0, \infty)$, and

(ii) has non-negative holomorphic bi-sectional and positive Ricci curvature, and

(iii) has $t \mathrm{R}(\cdot, t) \leq K$ for all $t>0$, and

(iv) $\sup _{(x, t) \in M \times(0, \infty)} t \mathrm{R}(x, t)$ is attained

must itself be an expanding Kähler gradient soliton. This result was generalised to the case of Ricci flow with non-negative curvature operator by B-L. Chen and X-P. Zhu in [10] (see Proposition 4.2 there). That is, if we have a solution to Ricci flow which satisfies the above with condition (ii) replaced by $(\tilde{i i})$ has non-negative curvature operator and positive Ricci curvature, then the conclusion is, the solution must be an expanding gradient soliton.

Hence, it is natural to look for solutions satisfying all or some of these conditions, when trying to construct expanding solitons with non-negative curvature operator.

Both of these theorems use the linear trace Harnack inequality of B. Chow and R. Hamilton (see [1]).

A pre-print of L. Ma 24 appeared after we had completed this paper. The pre-print contains a generalisation of the above result of B-L. Chen and X-P. Zhu [10]. In the paper [24, the condition $(\tilde{i i})$ above is replaced by the new ( $\tilde{i i})$ has non-negative Ricci curvature and is non-collapsed. The paper of L. Ma uses the $W_{+}$functional, which is a generalisation of Perleman's $W$ energy (see [28]). 
In the papers [16] and 25] the $W_{+}$functional is studied globally and locally. $W_{+}$ is monotone non-decreasing, and constant precisely on expanders (up to a shift in time). See for example Theorem 1.1 in [16] and also Corollary 6.9 of [25], where a similar result to that of [10] is proved using the $W_{+}$functional.

It is known that if an expanding soliton has bounded curvature and Ric $>0$, then $0<$ AVR $<\infty$. This was first proved by R. Hamilton for Ric $>0$ (see Proposition 9.46 in the book [12]). In the paper [7] this result was generalised to the case that the curvature is not necessarily bounded: see proposition 5.1 therein. Sharper estimates for expanding gradient solitons under weaker assumptions are also proved there. We refer the reader to that paper for more details. Similar estimates may also be found in Proposition 4.1 of the paper 8 .

Note that in our setting, we may assume that Ric $>0$ after isometrically splitting off a factor $\mathbb{R}^{m}$ (see Section 6 for more details). Hence, the assumption that the manifolds we consider have AVR $>0$ is natural.

Further examples of and estimates on expanding, steady and shrinking solitons on $\mathbb{C}^{m}$ are given in [15. In particular, they construct an example of a Ricci flow which starts as a shrinking soliton (for time less than zero), flows into a cone at time zero and then into a smooth expanding soliton (for time bigger than zero). They also include a discussion (with justification) on the desirable properties of a weak Ricci flow.

The splitting result that we prove in Appendix A is essentially derived from that of Hamilton in [19] (see also [5]).

Structure of the paper: In chapter three we fix some notation. In chapter four we prove a short time existence result for smooth, complete, non-collapsed Riemannian manifolds with non-negative and bounded curvature operator. In chapter five we show by a scaling argument that these conditions actually imply longtime existence if the asymptotic volume ratio is positive. Furthermore the asymptotic volume ratio for the so obtained solutions remains constant. By blowing down such a flow parabolically we prove in chapter six that we obtain a smooth limiting solution, which evolves out of the asymptotic cone at infinity of the initial manifold. We furthermore show that this solution actually is an expanding soliton. In Appendix A we give a proof of a splitting result, which has its origin in the de Rham Splitting Theorem. In Appendix B we recall an approximation result from V. Kapovitch/G.Perelman.

\section{Notation}

For a smooth Riemannian manifold $(M, g)$, and a family $(M, g(t))_{t \in[0, T)}$ of smooth Riemannian metrics, we use the notation

- $(M, d(g))$ is the metric space associated to the Riemannian manifold $(M, g)$, - $d \mu_{g}$ is the volume form of the Riemannian manifold $(M, g)$,

- $d(x, y, t)=\operatorname{dist}_{g(t)}(x, y)=d(g(t))(x, y)$ is the distance between $x$ and $y$ in $M$ with respect to the metric $g(t)$,

- ${ }^{g} B_{r}(x)$ is the ball of radius $r$ and centre $x$ measured with respect to $d(g)$,

- $B_{r}(x, t)$ is the ball of radius $r$ and centre $x$ measured with respect to $d(g(t))$,

- $\operatorname{vol}(\Omega, g)$ is the volume of $\Omega$ with respect to the metric $g$,

- $\operatorname{vol}\left({ }^{g} B_{r}(x)\right)=\operatorname{vol}\left({ }^{g} B_{r}(x), g\right)$,

- $\operatorname{vol}\left(B_{r}(x, t)\right)=\operatorname{vol}\left(B_{r}(x, t), g(t)\right)$,

- $\mathcal{R}(g)$ is the curvature operator of $g$, 
- $\operatorname{Riem}(g)$ is the curvature tensor of $g$,

- Ric(g) is the Ricci curvature tensor of $g$,

- $\mathrm{R}(g)$ is the scalar curvature of $g$,

- $\mathrm{R}(p, g)$ is the scalar curvature of the metric $g$ at the point $p$.

- If we write $B_{r}(x)$ resp. $\operatorname{vol}\left(B_{r}(x)\right)$ then we mean ${ }^{g} B_{r}(x)$ resp. $\operatorname{vol}\left({ }^{g} B_{r}(x)\right)$, where $g$ is a metric which will be clear from the context.

\section{Short time EXISTEnCE}

Let $\left(M^{n}, g_{0}\right)$ be any smooth, complete manifold with bounded curvature and without boundary. From the results of R. Hamilton [18] and W-X. Shi [29], we know that there exists a solution $(M, g(t))_{t \in[0, T)}$ to Ricci flow with $g(0)=g_{0}$ and $T \geq S\left(n, k_{0}\right)>0$ where $k_{0}:=\sup _{M}\left|\operatorname{Riem}\left(g_{0}\right)\right|$. That is: we can find a solution for a positive amount of time $T$ and $T$ is bounded from below by a constant depending on $k_{0}$ and $n$. The results of the paper 30] show that if the initial manifold is smooth, complete, without boundary and has non-negative bounded curvature operator and $\operatorname{vol}\left(B_{1}(x, 0)\right) \geq v_{0}>0$ for all $x \in M$, then the there exists a solution for a time interval $[0, T)$ where $T \geq S\left(n, v_{0}\right)$. Note the difference to the results of Hamilton and Shi: the lower bound on the length of the time interval of existence does not depend on the constant $k_{0}:=\sup _{M}\left|\operatorname{Riem}\left(g_{0}\right)\right|<\infty$. Some estimates on the evolving curvature were also proved in that paper. We state this result here, and give a proof using the results of $[30]$.

Theorem 4.1. Let $\left(M, g_{0}\right)$ be smooth, complete Riemannian manifold without boundary, with non-negative and bounded curvature operator. Assume also that the manifold is non-collapsed, that is

$$
\operatorname{vol}\left(B_{1}(x, 0)\right) \geq v_{0}>0 \quad \forall x \in M .
$$

Then there exist constants $T=T\left(n, v_{0}\right)>0$ and $K\left(n, v_{0}\right)$ and a solution to Ricci flow $(M, g(t))_{t \in[0, T)}$ which satisfies

$$
\begin{aligned}
& \left(a_{t}\right) \quad \mathcal{R}(g(t)) \geq 0 \\
& \left(b_{t}\right) \quad \operatorname{vol}\left(B_{1}(x, t)\right) \geq v_{0} / 2, \\
& \left(c_{t}\right) \sup _{M}|\operatorname{Riem}(g(t))| \leq K^{2} / t, \\
& \left(d_{t}\right) \quad d(p, q, s) \geq d(p, q, t) \geq d(p, q, s)-K(\sqrt{t}-\sqrt{s})
\end{aligned}
$$

for all $x, p, q \in M, 0<s \leq t \in[0, T)$, where $\mathcal{R}(g)$ is the curvature operator of $g$.

Proof. The proof follows from the results contained in the paper 30 and some other well known facts about Ricci flow. Using the result of [29], we obtain a maximal solution $(M, g(t))_{t \in\left[0, T_{\max }\right)}$ to Ricci flow with $g(0)=g_{0}$, where $T_{\max }>0$ and $\sup _{M}|\operatorname{Riem}(g(t))|<\infty$ for all $t \in\left[0, T_{\max }\right)$ and $\lim _{t} \tau_{\max } \sup _{M}|\operatorname{Riem}(g(t))|=\infty$ if $T_{\max }<\infty$. Also the curvature operator of the solution is non-negative at each time, since non-negative curvature operator is preserved for solutions with bounded curvature due to the maximum principle: see 21] and for example the argument in Lemma 5.1 of [30] (the argument there shows that the maximum principle is applicable to this non-compact setting, in view of the fact that the curvature is bounded. Maximum principles of this sort are well known: see for example [14] or [26]). Now the result follows essentially by following the proof of Theorem 6.1 in [31]. For convenience we sketch the argument here, and refer the reader to the 
proof there for more details. Let $\left[0, T_{M}\right)$ be the maximal time interval for which the flow exists and

$$
\inf _{x \in M} \operatorname{vol}\left(B_{1}(x, t)\right)>\frac{v_{0}}{2},
$$

for all $t \in\left[0, T_{M}\right)$. Using the maximum principle and standard ODE estimates, one shows easily that $T_{M}>0$ (see the proof of Theorem 7.1 in [30] for details). The aim is now to show that $T_{M} \geq S$ for some $S=S\left(n, V_{0}\right)>0$. From Lemma 4.3 of [30] we see that if $T_{M} \geq 1$ then the estimates $\left(a_{t}\right),\left(b_{t}\right)$ and $\left(c_{t}\right)$ are satisfied for all $t \leq 1$, and $\left(d_{t}\right)$ would then follow from Lemma 6.1 of [30], and hence we would be finished. So w.l.o.g. $T_{M} \leq 1$. From Lemma 4.3 of 30 once again,

$$
\mid \operatorname{Riem}\left(g(t) \mid \leq \frac{c_{0}\left(n, v_{0}\right)}{t}\right.
$$

for all $t \in\left(0, T_{M}\right)$ for some $c_{0}=c_{0}\left(n, v_{0}\right)<\infty$. First note that $\left(d_{t}\right)$ holds on the interval $\left(0, T_{M}\right)$ in view of Lemma 6.1 in 30 , and the fact that $\operatorname{Ric}(\mathrm{g}(\mathrm{t})) \geq 0$. Using Corollary 6.2 of [30], we see that there exists an $S=S\left(v_{0}, c_{0}\left(v_{0}, n\right)\right)=S\left(n, v_{0}\right)>0$, such that $\operatorname{vol}\left(B_{1}(x, t)\right)>2 v_{0} / 3$ for all $t \in\left[0, T_{M}\right) \cap[0, S)$. If $T_{M}<S$, then we obtain a contradiction to the definition of $T_{M}$ ( $T_{M}$ is the first time where the condition (4.3) is violated). Hence $T_{M} \geq S$. But then we may use Lemma 4.3, Lemma 6.1 of [30] to show that $\left(a_{t}\right),\left(b_{t}\right),\left(c_{t}\right)$ and $\left(d_{t}\right)$ are satisfied on $(0, S)$, as required.

Remark 4.2. Note that $T_{\max } \geq\left(U(n) / k_{0}\right)$ and $\sup _{M}|\operatorname{Riem}(g(t))| \leq k_{0} \tilde{k}(n)$ for all $t \leq\left(U(n) / k_{0}\right)$ for our solution, where $k_{0}:=\sup _{x \in M}\left|\operatorname{Riem}\left(g_{0}\right)\right|<\infty$ and $\tilde{k}(n), U(n)>0$ are constants. This is due to the fact that our solution is constructed by extending a Shi solution, and the solutions of Shi satisfy such estimates by scaling.

\section{LONG TIME EXISTENCE AND ESTimates}

The long time existence result follows essentially from scaling.

Theorem 5.1. Let $\left(M, g_{0}\right)$ be smooth, complete, without boundary, with nonnegative bounded curvature operator. Assume also that $\operatorname{AVR}\left(M, g_{0}\right)=: V_{0}>0$. Then there exists a solution to Ricci flow $(M, g(t))_{t \in[0, \infty)}$ with $g(0)=g_{0}$. Furthermore, the solution satisfies the following estimates.

$$
\begin{array}{cc}
\left(a_{t}\right) & \mathcal{R}(g(t)) \geq 0 \\
\left(b_{t}^{\prime}\right) & \operatorname{AVR}(M, g(t))=V_{0} \\
\left(c_{t}\right) & \sup _{M}|\operatorname{Riem}(g(t))| \leq \frac{K^{2}}{t}, \\
\left(d_{t}\right) & d(p, q, 0) \geq d(p, q, t) \geq d(p, q, s)-K(\sqrt{t}-\sqrt{s})
\end{array}
$$

for all $t \in[0, \infty)$ and $p, q \in M$, where $K=K\left(n, V_{0}\right)>0$ is a positive constant and $\mathcal{R}(g)$ is the curvature operator of $g$.

Proof. Let $c \in(0, \infty)$ and $\tilde{g}_{0}:=c g_{0}$. Then we still have $\sup _{M}\left|\operatorname{Riem}\left(\tilde{g}_{0}\right)\right|<\infty$ and $\operatorname{AVR}\left(M, \tilde{g}_{0}\right)=V_{0}>0$ as $\operatorname{AVR}(M, g)$ is a scale invariant quantity. From the Bishop-Gromov comparison principle, we have $\operatorname{vol}\left(\tilde{B}_{1}(x)\right) \geq V_{0}>0$ for all $x \in M$. Using the result above (Theorem [4.1), we obtain a solution $(M, \tilde{g}(t))_{t \in\left[0, T\left(n, V_{0}\right) \text { ) }\right.}$ satisfying $\tilde{g}(0)=\tilde{g}_{0}$ and the estimates $\left(a_{t}\right),\left(b_{t}\right),\left(c_{t}\right),\left(d_{t}\right)$ for all $t \in\left[0, T\left(n, V_{0}\right)\right)$. Setting $g(t):=(1 / c) \tilde{g}(c t)$, for $t \in[0,(T / c))$ we also obtain a solution to Ricci flow with bounded non-negative curvature operator, satisfying $g(0)=g_{0}$ and the estimates $\left(a_{t}\right),\left(c_{t}\right)$ and $\left(d_{t}\right)$ for all $t \in[0,(T / c))$, as $\left(a_{t}\right),\left(c_{t}\right),\left(d_{t}\right)$ are invariant 
under this scaling. Furthermore, by [32, Theorem 7] we have $\operatorname{AVR}(M, g(t))=V_{0}$, and thus $\operatorname{vol}\left(B_{r}(x, t)\right) \geq V_{0} r^{n}$ for all $r>0$. Now taking a sequence $c_{i} \rightarrow 0$ (in place of $c$ in the argument above), we obtain the result, in view of this estimate, $\left(a_{t}\right)$ and $\left(c_{t}\right)$, and the estimates of Shi and the compactness Theorem of Hamilton [20], see [21]. Note that in fact $\sup _{M}|\operatorname{Riem}(g(t))| \leq k_{0} \tilde{k}(n)$ for all $t \leq U(n) / k_{0}$, where $k_{0}:=\sup _{M}\left|\operatorname{Riem}\left(g_{0}\right)\right|$, in view of Remark 4.2. Additionally $\sup _{M}|\operatorname{Riem}(g(t))| \leq$ $\frac{K^{2} k_{0}}{U(n)}$ for all $t \geq U(n) / k_{0}$ in view of the scale invariant estimate $\left(c_{t}\right)$ and hence the results of Shi (see [21]) apply.

\section{Rescaling}

In this chapter we show that it is possible to scale down solutions of the type obtained in Theorem 5.1 to obtain an expanding soliton coming out of the asymptotic cone $\left(X, d_{X}\right)$ at infinity of $(M, h)$.

Proof of Theorem 1.2. We assume that $(M, h)$ is a smooth manifold with nonnegative, bounded curvature operator and positive asymptotic volume ratio $V_{0}:=$ $\operatorname{AVR}(M, h)>0$. Now let $c_{i} \rightarrow 0$ be a sequence of positive numbers, converging to zero. Then $\left(M, c_{i} h, p_{0}\right)$ converges in the pointed Gromov-Hausdorff sense to the metric cone $\left(X, d_{X}, 0\right)$. By Theorem 5.1 there exists a Ricci flow $(M, g(t))_{t \in[0, \infty)}$ with $g(0)=h$ which satisfies $\left(a_{t}\right),\left(c_{t}\right)$ and $\operatorname{AVR}(M, g(t))=\operatorname{AVR}(M, g(0))$. By Hamilton's Harnack estimate, see for example equation 10.46 of Chapter $10, \S 4$ of [12], we have

$$
\frac{\partial}{\partial t}(t \mathrm{R}(p, g(t))) \geq 0
$$

for all $t \in[0, \infty)$ for all $p \in M$. We define the scaled Ricci flows $\left(M, g^{i}(t)\right)_{t \in[0, \infty)}$ by

$$
g^{i}(t):=c_{i} g\left(t / c_{i}\right)
$$

Note that these flows still satisfy $\left(a_{t}\right),\left(c_{t}\right)$ and $\operatorname{AVR}\left(M, g^{i}(t)\right)=\operatorname{AVR}(M, h)$ and we have a uniform lower bound for the injectivity radius for times $t \in[\delta, \infty), \delta>0$, since the curvature is uniformly bounded and the volume of balls is uniformly bounded from below on such time intervals. Hence, we may take a pointed limit of the flows $\left(M, g^{i}(t), p_{0}\right)_{t \in(0, \infty)}$ to obtain a smooth Ricci flow $\left(\tilde{X}, \tilde{g}(t), \tilde{x}_{0}\right)_{t \in(0, \infty)}$ with

$$
\tilde{V}_{0}:=\operatorname{AVR}(\tilde{X}, \tilde{g}(t)) \geq \operatorname{AVR}(M, h)=V_{0}>0
$$

for all $t \in(0, \infty)$. $\tilde{V}_{0}$ is a constant by [32, Theorem 7]. Note that we also have the following estimates: $d_{X}(\cdot, \cdot) \geq d(\tilde{g}(t))(\cdot, \cdot) \geq d_{X}(\cdot, \cdot)-K \sqrt{t}$ where $\left(X, d_{X}, 0\right)$ is the asymptotic cone at infinity of $(M, h)$. These estimates follow after taking a limit of the estimates $\left(d_{t}\right) d\left(c_{i} h\right)(\cdot, \cdot) \geq d\left(g^{i}(t)\right)(\cdot, \cdot) \geq d\left(c_{i} h\right)(\cdot, \cdot)-K \sqrt{t}$, which hold by construction of our solution. In particular we see that $\left(\tilde{X}, \tilde{g}(t), \tilde{x}_{0}\right)$ converges in the pointed Gromov-Hausdorff sense to $\left(X, d_{X}, 0\right)$ as $t \rightarrow 0$.

A result of Cheeger and Colding (see Theorem 5.4 of 9 ) gives that volume is continuous under the Gromov-Hausdorff limit of non-collapsing spaces with Ricci curvature bounded below. Thus since $\left(\tilde{X}, \tilde{g}(t), \tilde{x}_{0}\right)$ converges to the asymptotic cone at infinity $\left(X, d_{X}, 0\right)$ of $\left(M, h, p_{0}\right)$ as $t \rightarrow 0$, and the Bishop-Gromov volume comparison principle holds, we have

$$
\tilde{V}_{0} \leq \operatorname{AVR}\left(X, d_{X}\right)=\operatorname{AVR}(M, h)=V_{0},
$$


and thus $\tilde{V}_{0}=V_{0}$. By (6.1), we also have $\frac{\partial}{\partial t}\left(t \mathrm{R}\left(p, g^{i}(t)\right)\right) \geq 0$ for all $t \in[0, \infty)$ and all $p \in M$. For $p \in M$ define $S(p):=\lim _{t \rightarrow \infty} t \mathrm{R}(p, g(t))$, which is a well defined and positive real (non-infinite) number by (6.1) and $\left(c_{t}\right)$. Since this quantity is scale-invariant it follows that

$$
\lim _{i \rightarrow \infty} t_{0} \mathrm{R}\left(p, g^{i}\left(t_{0}\right)\right)=S(p)
$$

for any fixed $t_{0}>0$. Note that the convergence of $\left(M, g^{i}(t), p_{0}\right) \rightarrow\left(\tilde{X}, \tilde{g}(t), \tilde{x}_{0}\right)$ is smooth on compact sets contained in $(0, \infty) \times \tilde{X}$, and $p_{0}$ is mapped by the diffeomorphisms involved in the pointed Hamilton-Cheeger-Gromov convergence onto $\tilde{x}_{0}$, thus we have

$$
t \mathrm{R}\left(\tilde{x}_{0}, \tilde{g}(t)\right)=S\left(p_{0}\right)
$$

for all $t>0$. Recall that the evolution equation for the Ricci curvature is given by

$$
\frac{\partial}{\partial t} \operatorname{Ric}^{\mathrm{i}}{ }_{\mathrm{j}}=\Delta \operatorname{Ric}_{\mathrm{j}}^{\mathrm{i}}+2 \operatorname{Ric}_{\mathrm{s}}^{\mathrm{r}} \operatorname{Riem}_{\mathrm{rj}}^{\mathrm{i}}{ }^{\mathrm{s}} .
$$

Now note, that if $\operatorname{Ric}(\mathrm{y}, \tilde{\mathrm{g}}(\mathrm{t}))(\mathrm{Y}, \mathrm{Y})=0$ for some $Y \in T_{y} \tilde{X}$ then we have in view of the de Rham Decomposition Theorem (see Appendix $₫$ with $h$ of the Decomposition Theorem equal to Ric) a splitting, $(\tilde{X}, \tilde{g}(t))=(L \times \Omega, h \oplus l(t))$ where $(L, h)$ has zero curvature operator and $(\Omega, l(t))$ has positive Ricci curvature (here we use that $\operatorname{Ric}(\mathrm{Y}, \mathrm{Y})=0$ implies $\sec (Y, V)=0$ for all $V$ in view of the fact that $\mathcal{R} \geq 0)$. In fact $(L, h)=\left(\mathbb{R}^{k}, h\right)$ where $h$ is the standard metric. This may be seen as follows. If $(L, h)$ is not $\left(\mathbb{R}^{k}, h\right)$, then the first fundamental group of $(L, h)$ is non-trivial. This would imply in particular that the first fundamental group of $(L \times \Omega, h \oplus l(t))=$ $(\tilde{X}, \tilde{g}(t))$ is also non-trivial. Using the same argument given in Theorem 9.1 of [30] (see Lemma 6.2 in this paper for some comments thereon), we see that $\left(\tilde{X}, \tilde{g}(t), \tilde{x}_{0}\right)$ is homeomorphic to $\left(X, d_{X}, 0\right)$, and hence $\left(X, d_{X}\right)$ has non-trivial first fundamental group. But as explained at the beginning of this paper $\left(X, d_{X}\right)$ is a cone over a standard sphere. In particular $\left(X, d_{X}\right)$ is homeomorphic to $\mathbb{R}^{n}$. Hence $\left(X, d_{X}\right)$ has trivial first fundamental group which leads to a contradiction.

Hence, we may write $(\tilde{X}, \tilde{g}(t))=\left(\mathbb{R}^{k} \times \Omega, h \oplus l(t)\right)$ where $(\Omega, l(t))$ is a solution to the Ricci flow satisfying $\left(a_{t}\right),\left(c_{t}\right)$ and $\operatorname{Ric}(\mathrm{l}(\mathrm{t}))>0$ for all $t>0$. Using Fubini's theorem, it is easy to see that the asymptotic volume ratio of $l(t)$ is given by $\left(\omega_{n-k} / \omega_{n}\right) \tilde{V}_{0}>0$, where $\omega_{m}$ is the volume of the $m$-dimensional Euclidean unit ball.

We show in the following that $(\Omega, l(t))_{t \in(0, \infty)}$ is a gradient expanding soliton, generated by some smooth function $f$. Hence $(\tilde{X}, \tilde{g}(t))=\left(\mathbb{R}^{k} \times \Omega, h \oplus l(t)\right)$ is a gradient expanding soliton: $\nabla^{2} f(t)-\operatorname{Ric}(\mathrm{l}(\mathrm{t}))-(1 /(2 \mathrm{t})) \mathrm{l}(\mathrm{t})=0$ on $\Omega$ and $\nabla^{2} v(t)-$ $\operatorname{Ric}(\mathrm{h})-(1 /(2 \mathrm{t})) \mathrm{h}=0$ on $\mathbb{R}^{k}$ where $v(x, t)=\frac{|x|^{2}}{4 t}$, and hence $\nabla^{2} \tilde{f}(t)-\operatorname{Ric}(\tilde{\mathrm{g}}(\mathrm{t}))-$ $(1 /(2 \mathrm{t})) \tilde{\mathrm{g}}(\mathrm{t})=0$ for all $t>0$ on $\tilde{X}$ with $\tilde{f}(x, y, t)=f(y, t)+v(x, t)$ for $(x, y) \in$ $\left(\mathbb{R}^{k} \times \Omega\right)$.

For simplicity let us denote $l(t)$ again by $\tilde{g}(t)$, i.e. we assume that $k=0$.

Remark 6.1. (i) After completing this paper we noticed that we could use the proof of Proposition 12 of the paper of $S$. Brendle [1] at this point to show that $(X, \tilde{g}(t))$ is an expanding gradient soliton. We include here our original proof which follows the lines of that given in 12 .

(ii) In 12] and 10, it is assumed that $t \mathrm{R}(\cdot, t)$ achieves its maximum somewhere in order to conclude that the solution is a soliton. We make no such assumption. We 
show that $\nabla R\left(\tilde{x}_{0}\right)=0$ in view of the fact that $\frac{\partial}{\partial t}\left(t R\left(t, \tilde{x}_{0}\right)\right)=0$, and then argue as in 12 and [10].

For the rest of this argument we work with the Riemannian metrics $\tilde{g}(t)$. For ease of reading we introduce the notation $\mathrm{R}(x, t):=\mathrm{R}(x, \tilde{g}(t))$, $\operatorname{Ric}(\mathrm{x}, \mathrm{t}):=\operatorname{Ric}(\tilde{\mathrm{g}}(\mathrm{t}))(\mathrm{x})$ and so on. All metrics and covariant derivatives are taken with respect to the metrics $\tilde{g}(t)$. We assume that $\tilde{g}_{i j}=\delta_{i j}$ at points where we calculate, and indices that appear twice are summed. We saw before that we may assume that $\frac{\partial}{\partial t}\left(t \mathrm{R}\left(\tilde{x}_{0}, t\right)\right)=$ 0 . But then

$$
0=\frac{\partial}{\partial t}\left(t \mathrm{R}\left(\tilde{x}_{0}, t\right)\right)_{t=1}=\Delta \mathrm{R}\left(\tilde{x}_{0}, 1\right)+2|\operatorname{Ric}|^{2}\left(\tilde{\mathrm{x}}_{0}, 1\right)+\mathrm{R}\left(\tilde{\mathrm{x}}_{0}, 1\right)
$$

By Theorem 10.46 in [12], with $v_{i j}=\mathrm{Ric}_{\mathrm{ij}}$, we have

$$
Z(Y):=\nabla_{i} \nabla_{j} \operatorname{Ric}_{\mathrm{ij}}=\mid \operatorname{Ric}^{2}+2\left(\nabla_{\mathrm{j}} \operatorname{Ric}_{\mathrm{ij}}\right) \mathrm{Y}_{\mathrm{i}}+\operatorname{Ric}_{\mathrm{ij}} \mathrm{Y}_{\mathrm{i}} \mathrm{Y}_{\mathrm{j}}+\frac{\mathrm{R}}{2 \mathrm{t}} \geq 0,
$$

for any tangent vector $Y$. In particular for

we see that

$$
Y=-\left(\operatorname{Ric}^{-1}\right)^{\mathrm{ji}} \operatorname{div}(\operatorname{Ric})_{\mathrm{j}} \frac{\partial}{\partial \mathrm{x}^{\mathrm{i}}}=-(1 / 2)\left(\operatorname{Ric}^{-1}\right)^{\mathrm{ji}} \nabla_{\mathrm{j}} \mathrm{R} \frac{\partial}{\partial \mathrm{x}^{\mathrm{i}}},
$$

$$
\begin{aligned}
Z(Y)= & (1 / 2) \Delta \mathrm{R}+\mid \mathrm{Ric}^{2}-(1 / 2)\left(\mathrm{Ric}^{-1}\right)^{\mathrm{ij}}\left(\nabla_{\mathrm{i}} \mathrm{R}\right)\left(\nabla_{\mathrm{j}} \mathrm{R}\right) \\
& +(1 / 4) \mathrm{Ric}_{\mathrm{ij}}\left(\mathrm{Ric}^{-1}\right)^{\mathrm{si}} \nabla_{\mathrm{s}} \mathrm{R}\left(\mathrm{Ric}^{-1}\right)^{\mathrm{jk}} \nabla_{\mathrm{k}} \mathrm{R}+\frac{\mathrm{R}}{2 \mathrm{t}} \\
= & (1 / 2)\left(\Delta \mathrm{R}+2|\mathrm{Ric}|^{2}-(1 / 2)\left(\operatorname{Ric}^{-1}\right)^{\mathrm{ij}}\left(\nabla_{\mathrm{i}} \mathrm{R}\right)\left(\nabla_{\mathrm{j}} \mathrm{R}\right)+\frac{\mathrm{R}}{\mathrm{t}}\right) \geq 0 .
\end{aligned}
$$

Using (6.3), we have

$$
0 \leq Z(Y)\left(\tilde{x}_{0}, 1\right)=-(1 / 4)\left(\operatorname{Ric}^{-1}\right)^{\mathrm{ij}}\left(\nabla_{\mathrm{i}} \mathrm{R}\right)\left(\nabla_{\mathrm{j}} \mathrm{R}\right)\left(\tilde{\mathrm{x}}_{0}, 1\right)
$$

and hence $\nabla \mathrm{R}\left(\tilde{x}_{0}, 1\right)=0$. This implies that

$$
Z(Y)\left(\tilde{x}_{0}, 1\right)=0
$$

which is a global minimum for $Z(Y)$. Now we use the evolution equation for $Z(Y)$, which is given by equation (10.73) in [12. It implies in particular that

$$
\frac{\partial}{\partial t} Z(Y) \geq \Delta Z(Y)
$$

and hence by the strong maximum principle, we must have $Z(Y)=0$ everywhere. By looking once again at the equation (10.73) in [12] and using the matrix Harnack inequality and the fact that $Z(Y)=0$, we get

$$
\frac{\partial}{\partial t} Z(Y) \geq 2 v_{i j}\left(\nabla_{k} Y_{i}-\operatorname{Ric}_{\mathrm{ik}}-(1 /(2 \mathrm{t})) \delta_{\mathrm{ik}}\right)\left(\nabla_{\mathrm{k}} \mathrm{Y}_{\mathrm{j}}-\mathrm{Ric}_{\mathrm{jk}}-(1 /(2 \mathrm{t})) \delta_{\mathrm{jk}}\right) .
$$

If at some point in space and time we have $\nabla Y-\operatorname{Ric}-(1 /(2 \mathrm{t})) \tilde{\mathrm{g}} \neq 0$ as a tensor, then we get $2 v_{i j}\left(\nabla_{k} Y_{i}-\mathrm{R}_{i k}-(1 /(2 t)) \delta_{i k}\right)\left(\nabla_{k} Y_{j}-\mathrm{R}_{j k}-(1 /(2 t)) \delta_{j k}\right)>0$ which would imply that $\frac{\partial}{\partial t} Z(Y)>0$ at this point in space and time. This in turn would imply that there are points in space and time with $Z(Y)>0$, which is a contradiction. Hence $\nabla Y-$ Ric $-(1 /(2 \mathrm{t})) \tilde{\mathrm{g}}=0$, which yields that $\tilde{g}(t)$ is an expanding gradient soliton.

For completeness we include the following Lemma, whose statement and proof appeared in the proof we just gave. 
FELIX SCHULZE AND MILES SIMON

Lemma 6.2. Let $\left(\tilde{X}, \tilde{g}(t), \tilde{x}_{0}\right)_{t \in(0, \infty)}:=\lim _{i \rightarrow \infty}\left(M, g_{i}(t), p_{0}\right)_{t \in(0, \infty)}$ be the solution obtained above, and $\left(X, d_{X}, 0\right)$ be the pointed Gromov-Hausdorff limit of $\left(M, c_{i} h, p_{0}\right)=\left(M, g_{i}(0), p_{0}\right),\left(c_{i} \rightarrow 0\right)$. Then $\left(\tilde{X}, d(\tilde{g}(t)), \tilde{x}_{0}\right) \rightarrow\left(X, d_{X}, 0\right)$ in the pointed Gromov-Hausdorff sense as $t \rightarrow 0$. That is, the solution flows out of the cone $\left(X, d_{X}, 0\right)$. Furthermore, $\tilde{X}$ is homeomorphic to $X$ which is homeomorphic to $\mathbb{R}^{n}$.

Proof. (We repeat the proof given above). Using the same argument given in Theorem 9.1 of [30], we see that $\left(\tilde{X}, \tilde{g}(t), \tilde{x}_{0}\right)$ is homeomorphic to $\left(X, d_{X}, 0\right)$ ( note that in the argument there, $U$ and $V$ should be bounded open sets: this is sufficient to conclude that the topologies are the same, since any open set can be written as the union of bounded open sets in a metric space). But as explained at the beginning of this paper $\left(X, d_{X}\right)$ is a cone over a sphere, where the topology of the sphere is the same as that of the standard sphere. In particular $\left(X, d_{X}\right)$ is homeomorphic to $\mathbb{R}^{n}$.

\section{ApPendix A. DE Rham SPLitTing}

In this appendix, we explain some known splitting results, which follow from the de Rham Splitting Theorem. We give proofs for the reader's convenience. We follow essentially the argument given in the proof of Theorem 2.1 of [5] which follows closely that of Lemma 8.2/Theorem 8.3 of [19]. For a two tensor $\beta_{i j}$ we let $\beta_{j}^{i}:=g^{i l} \beta_{l j}$, i.e. this defines an endomorphism from each tangent space into itself.

Theorem A.1. Let $h(t)_{t \in[0, T)}$ be a smooth (in space and time) bounded family of symmetric two tensors defined on a simply connected complete manifold $M^{n}$ without boundary, satisfying the evolution equation

$$
\frac{\partial}{\partial t} h^{i}{ }_{j}={ }^{g(t)} \Delta h^{i}{ }_{j}+\phi^{i}{ }_{j}
$$

where $h(x, t), \phi(x, t) \geq 0$ for all $(x, t) \in M \times[0, T)$ in the sense of matrices. We also assume $g$ is a smooth family of metrics (in space and time) satisfying ${ }^{g_{0}}\left|D^{(i, j)} g\right|+$ ${ }^{g_{0}}\left|D^{(i, j)} h\right| \leq k(i, j)<\infty$ everywhere, where $i, j \in \mathbb{N}$ and $D^{(i, j)}$ refers to taking $i$ time derivatives and $j$ covariant derivatives with respect to $g_{0}$, and $k(i, j) \in \mathbb{R}$ are constants. Then for all $x \in M, t>0$, the null space of $h(x, t)$ is invariant under parallel translation and constant in time. There is a splitting, $(M, g(t))=$ $(N \times P, r(t) \oplus l(t))$, where $r, l$ are smooth families of Riemannian metrics such that $h>0$ on $N$ (as a two tensor), and $h=0$ on $P$.

Proof. Let $0 \leq \sigma_{1}(x, t) \leq \sigma_{2}(x, t) \leq \ldots \leq \sigma_{n}(x, t)$ be the eigenvalues of $h(x, t)$ and $\left\{e_{1}(x, t)\right\}$ orthonormal eigenvectors. Assume that $\sigma_{1}\left(x_{0}, t_{0}\right)+\sigma_{2}\left(x_{0}, t_{0}\right)+\ldots+$ $\sigma_{k}\left(x_{0}, t_{0}\right)>0$ at some point $x_{0}$ and some time $t_{0}$. Define a smooth function $\eta_{t_{0}}: M \rightarrow \mathbb{R}_{0}^{+}$which is positive at $x_{0}$ and zero outside of $B_{1}\left(x_{0}, 0\right)$ (the ball in $M$ of radius one with respect to $\left.g_{0}\right)$, and satisfies $\sigma_{1}\left(\cdot, t_{0}\right)+\sigma_{2}\left(\cdot, t_{0}\right)+\ldots+\sigma_{k}\left(\cdot, t_{0}\right)>\eta_{t_{0}}(\cdot)$. Solve the Dirichlet problem:

$$
\begin{aligned}
& \frac{\partial}{\partial t} \eta_{i}={ }^{g(t)} \Delta \eta_{i} \\
& \left.\eta_{i}(\cdot, t)\right|_{\partial B_{i}\left(x_{0}, 0\right)}=0 \quad \forall t \in\left[t_{0}, T\right) \\
& \eta_{i}\left(\cdot, t_{0}\right)=\eta_{t_{0}}(\cdot)
\end{aligned}
$$


Using the estimates for $g$ we see that the solutions exist for all time and satisfy interior estimates independent of $i$ (see for example Theorem 10.1, chapter IV, $\S 10$ in [23]). Thus we may take a subsequence to obtain a smooth solution $\eta$ : $M \times\left[t_{0}, T\right) \rightarrow \mathbb{R}$ of the equation

$$
\begin{aligned}
& \frac{\partial}{\partial t} \eta={ }^{g(t)} \Delta \eta \\
& \eta\left(\cdot, t_{0}\right)=\eta_{t_{0}}(\cdot) .
\end{aligned}
$$

From the strong maximum principle, $\eta(\cdot, t)>0$ for all $t>t_{0}$. Also, the construction and the estimates on $g$ guarantee that $\sup _{\left(M \backslash B_{i}\left(x_{0}, 0\right)\right) \times\left[t_{0}, S\right]}|\eta(\cdot, t)| \rightarrow 0$ as $i \rightarrow \infty$. for all $S<T$. We claim that $\sigma_{1}(\cdot, t)+\ldots+\sigma_{k}(\cdot, t) \geq e^{-a t} \eta(\cdot, t)$ for all $t \geq t_{0}$. One proves first, that $\sigma_{1}(\cdot, t)+\ldots+\sigma_{k}(\cdot, t)-e^{-a t} \eta(\cdot, t)+\varepsilon e^{\rho^{2}(\cdot, t)(1+a t)+a t} \geq 0$ for arbitrary small $\varepsilon>0$ and an appropriately chosen constant $a$, where here $\rho(x, t)=\operatorname{dist}\left(x, x_{0}, t\right)(a>0$ does not depend on $\varepsilon: a$ depends on the constants in the statement of the Theorem). This is done by using the maximum principle. See for example the argument in the proof of Lemma 5.1 in [30] for details. Now let $\varepsilon$ go to zero. This implies $\sigma_{1}(\cdot, t)+\ldots+\sigma_{k}(\cdot, t) \geq \eta(\cdot, t)$ for all $t \geq t_{0}$ and hence $\sigma_{1}(\cdot, t)+\ldots+\sigma_{k}(\cdot, t)>0$ for all $t>t_{0}$. Thus

$$
\operatorname{dim}(\operatorname{null}(h(x, t)))=\max \left\{i \in\{0, \ldots, n\} \mid \sigma_{1}(x, t)+\ldots+\sigma_{i}(x, t)=0\right\}
$$

is independent of $x \in M$ for all $t>t_{0}$ and a decreasing function in time. Hence $\operatorname{rank}(h(x, t))$ is constant in space and time for some short time interval $t_{0}<t<$ $t_{0}+\delta$ for any $t_{0} \in[0, T)$. Now we let $v$ be a smooth vector field in space and time lying in the null space of $h$ (at each point in space and time). We can always construct such sections which have length one in a small neighbourhood, by defining it locally smoothly, and then multiplying by a cut-off function. We follow closely the proof of Lemma 8.2 of Hamilton ([19]) and Theorem 2.1 of [5]. In the following we use the notation $\nabla$ and $\Delta$ to refer to ${ }^{g(t)} \nabla$ and ${ }^{g(t)} \Delta$. Using $h(v, v) \equiv 0$ we get

$$
\begin{aligned}
0=\frac{\partial}{\partial t}(h(v, v)) & =\left(\frac{\partial}{\partial t} h^{i}{ }_{j}\right) v_{i} v^{j}+h^{i}{ }_{j}\left(\left(\frac{\partial}{\partial t} v_{i}\right) v^{j}+v_{i}\left(\frac{\partial}{\partial t} v^{j}\right)\right) \\
& =\left(\frac{\partial}{\partial t} h^{i}{ }_{j}\right) v^{i} v^{j},
\end{aligned}
$$

since $h^{i}{ }_{j} v_{i}=0$ and $h^{i}{ }_{j} v^{j}=0$. Furthermore, since $h^{i}{ }_{j} v_{i} v^{j} \equiv 0$ we get

$$
\begin{aligned}
0= & \Delta\left(h^{i}{ }_{j} v_{i} v^{j}\right) \\
= & (\Delta h)^{i}{ }_{j} v_{i} v^{j}+2 \Delta\left(v_{i}\right) h^{i}{ }_{j} v^{j} \\
& +4 g^{k l} v^{j} \nabla_{k} h^{i}{ }_{j} \nabla_{l} v_{i}+2 g^{k l} h^{i}{ }_{j} \nabla_{k} v^{j} \nabla_{l} v_{i}
\end{aligned}
$$

The term $2 \Delta\left(v_{i}\right) h^{i}{ }_{j} v^{j}$ is once again zero, since $h^{i}{ }_{j} v^{j}=0$. Using this, (A.5), (A.4) and the evolution equation for $h$ we get

$$
\begin{aligned}
0 & =\left(\frac{\partial}{\partial t}\left(h^{i}{ }_{j}\right)-(\Delta h)_{j}^{i}-\phi^{i}{ }_{j}\right)\left(v_{i} v^{j}\right) \\
& =4 g^{k l} v^{j} \nabla_{k} h_{j}^{i} \nabla_{l} v_{i}+2 g^{k l} h^{i}{ }_{j} \nabla_{k} v^{j} \nabla_{l} v_{i}-\phi_{j}^{i} v_{i} v^{j}
\end{aligned}
$$

Now use

to conclude

$$
v^{j} \nabla_{k} h_{j}^{i}=\nabla_{k}\left(v^{j} h^{i}{ }_{j}\right)-h_{j}^{i} \nabla_{k} v^{j}=-h_{j}^{i} \nabla_{k} v^{j}
$$

$$
2 g^{k l} h^{i}{ }_{j} \nabla_{k} v^{j} \nabla_{l} v_{i}+\phi^{i}{ }_{j} v_{i} v^{j}=0
$$


Since $\phi(v, v) \geq 0$ (and $h \geq 0$ ) we see that $\phi^{i}{ }_{j} v_{i} v^{j}=0$. That is, $v$ is also in the null space of $\phi$. But then, (A.7) shows that $X_{R}(x, t):=\nabla_{R} v(x, t)$ is in the null space of $h$ for any vector $R \in T_{x} M$ (choose orthonormal coordinates at $x$ at time $t$, so that $\frac{\partial}{\partial x^{1}}(x):=R /\|R\|_{g(x, t)}$ and use this in equation (A.7)). This shows that the null space of $h$ is invariant under parallel transport for each fixed time, as explained in the following for the readers convenience:

Let $v_{1}(x), \ldots, v_{k}(x)$ be a smooth o.n. basis for $\operatorname{null}(h(x, t))$ in a small spatial neighbourhood of $x_{0}$, and extend this to a smooth family $v_{1}, \ldots, v_{n}$ of vectors which is an o.n. basis everywhere in a small spatial neighbourhood of $x_{0}$. Let $X_{0} \in T_{x_{0}} M$ satisfy $g\left(X_{0}, v_{i}\left(x_{0}\right)\right)=0$ for all $i \in\{k+1, \ldots, n\}$ and let $\gamma:[0,1] \rightarrow M$ be any smooth curve, starting in $x_{0}$ and whose image is contained in the neighbourhood of $x_{0}$ in question. Then parallel transport $X_{0}$ along $\gamma$. Call this vector field $X$. Write $X(\tau)=\sum_{i=1}^{n} X^{i}(\tau) v_{i}(\gamma(\tau))$. We claim $X(\tau)=\sum_{i=0}^{k} X^{i}(\tau) v_{i}(\gamma(\tau))$. Let $X^{\top}(\tau)=\sum_{i=1}^{k} X^{i}(\tau) v_{i}(\gamma(\tau))$, and $X^{\perp}(\tau)=\sum_{i=k+1}^{n} X^{i}(\tau) v_{i}(\gamma(\tau))$. First note that for $i \in\{1, \ldots, k\}$, and $V$ the tangent vector field along $\gamma$ :

$$
g\left(\nabla_{V}\left(X^{\perp}\right), v_{i}\right)=V\left(g\left(X^{\perp}, v_{i}\right)\right)-g\left(X^{\perp}, \nabla_{V} v_{i}\right)=0
$$

in view of $\nabla_{V} v_{i} \in \operatorname{span}\left\{v_{1}, \ldots, v_{k}\right\}$ and $X^{\perp} \in \operatorname{span}\left\{v_{k+1}, \ldots, v_{n}\right\}$. Furthermore, for $j \in\{k+1, \ldots, n\}$ we have

$$
\begin{aligned}
g\left(\nabla_{V}\left(X^{\perp}\right), v_{j}\right) & =g\left(\nabla_{V}\left(X-X^{\top}\right), v_{j}\right) \\
& =-g\left(\nabla_{V}\left(X^{\top}\right), v_{j}\right) \\
& =-g\left(\sum_{i=1}^{k} V\left(X^{i}\right) v_{i}, v_{j}\right)-g\left(\sum_{i=1}^{k} X^{i} \nabla_{V} v_{i}, v_{j}\right) \\
& =-\sum_{i=1}^{k} X^{i} g\left(\nabla_{V} v_{i}, v_{j}\right) \\
& =0
\end{aligned}
$$

in view of the fact that $\nabla_{V} v_{i} \in \operatorname{span}\left\{v_{1}, \ldots, v_{k}\right\}$. Hence $X^{\perp}$ is also parallel along $\gamma$. Since $X^{\perp}(0)=0$ we have $X^{\perp} \equiv 0$.

We have also shown that $\operatorname{null}(h) \subseteq \operatorname{null}(\phi)$. Let $v\left(x_{0}, s\right)$ for $s \in(t, t+\delta)$ be smoothly dependent on time, and $v\left(x_{0}, s\right) \in \operatorname{null}\left(h\left(x_{0}, s\right)\right)$ for each $s \in(t, t+$ $\delta)$. Extend this vector at each time $s \in(t, t+\delta)$ by parallel transport along geodesics emanating from $x_{0}$ to obtain a local smooth vector field $v(\cdot, \cdot)$ which satisfies $v(x, s) \in \operatorname{null}(h(x, s))$ for all $x$ (in a small ball) and all $s \in(t, t+\delta)$. In particular,

$$
\nabla_{i} v \in \operatorname{null}(h(x, s)) \text { and } \Delta v(x, s)=\left(g^{k l} \nabla_{k} \nabla_{l} v\right)(x, s) \in \operatorname{null}(h(x, s)) .
$$

Since $\nabla_{i} v\left(x_{0}, s\right)=0$ we can compute

$$
\begin{aligned}
0=\frac{\partial}{\partial t}\left(h^{i}{ }_{j} v_{i}\right) & =\left(h^{i}{ }_{j} \frac{\partial}{\partial t} v_{i}\right)+\left(v_{i}(\Delta h)^{i}{ }_{j}\right)+v_{i} \phi^{i}{ }_{j} \\
& =h^{i}{ }_{j} \frac{\partial}{\partial t} v_{i}+\Delta\left(v_{i} h^{i}{ }_{j}\right)+v_{i} \phi^{i}{ }_{j} \\
& =h^{i}{ }_{j} \frac{\partial}{\partial t} v_{i}
\end{aligned}
$$


where we have used that $v \in \operatorname{null}(\phi)$. Hence $\frac{\partial}{\partial t} v\left(x_{0}, s\right) \in \operatorname{null}\left(h\left(x_{0}, s\right)\right)$. Assume that at time $s_{0}$ we have $\operatorname{null}\left(h\left(x_{0}, s_{0}\right)\right)=\mathbb{R}^{k} \subseteq \mathbb{R}^{n}=T_{x_{0}} M$ and let $\left\{e_{1}(t), \ldots, e_{n}(t)\right\}$ be a smooth (in time) o.n. basis of vectors with $\left\{e_{1}(t), \ldots, e_{k}(t)\right\}$ a smooth (in time) o.n. basis of vectors of $\operatorname{null}\left(h\left(x_{0}, t\right)\right)$. Let $e_{i}^{l}(t):=\left\langle e_{i}(t), e^{l}(0)\right\rangle$, where $\left\{e^{1}(0), \ldots, e^{n}(0)\right\}$ refer to the standard basis vectors of $\mathbb{R}^{n}$ and $\langle\cdot, \cdot\rangle$ is the standard inner product on $\mathbb{R}^{n} \cdot \frac{\partial}{\partial t} e_{i}(t) \in \operatorname{null}(h(t))$ for all $i \in\{1, \ldots, k\}$ implies $\frac{\partial}{\partial t} e_{i}(t)=\sum_{j=1}^{k} a_{i}^{j}(t) e_{j}(t)$ for some smooth functions $a_{i}^{j}:[0, \infty) \rightarrow \mathbb{R}, i, j \in\{1, \ldots, k\}$. Then we have a system of ODEs $(l \in\{1, \ldots, n\}, i \in\{1, \ldots, k\})$

$$
\begin{aligned}
& \frac{\partial}{\partial t} e_{i}^{l}(t)=\sum_{j=1}^{k} a_{i}^{j}(t) e_{j}^{l}(t) \\
& e_{i}^{l}(0)=\delta_{i}^{l} .
\end{aligned}
$$

By assuming $e_{j}^{l}(t)=0$ for all $l \geq k+1$ we still have a solvable system, and hence the solution satisfies (by uniqueness) $e_{j}^{l}(t)=0$ for all $l \geq k+1$. That is $\left\{e_{1}(t), \ldots, e_{k}(t)\right\}$ remains in $\mathbb{R}^{k}$.

$\operatorname{null}\left(h\left(x_{0}, t\right)\right)$ is a space which is invariant under parallel transport (from the argument above). Hence the de Rham splitting theorem (see [13) says, $M$ splits isometrically at time $s$ as $N(s) \oplus P(s)$ where $h(\cdot, s)=0$ on $P(s)$ and $h(\cdot, s)>0$ on $N(s)$. We can do this it every time $s$. But the second part of the argument shows that $N(s)=N\left(s_{0}\right)$ for all $s$ and $P(s)=P\left(s_{0}\right)$ for all $s$.

\section{Appendix B. An approximation result by V. Kapovitch/G. Perelman.}

Let $\left(M_{i}, d_{i}, p_{0}\right)$ be a non-collapsing sequence of non-negatively curved $n$-dimensional, smooth, complete manifolds without boundary such that $\left(M_{i}^{n}, d_{i}, p_{0}\right) \rightarrow$ $\left(X, d_{X}, 0\right)$ as $i \rightarrow \infty$ (in the GH sense) where $X=C V$ is an Euclidean cone with non-negative curvature over the metric space $\left(V, d_{V}\right)$ (with sectional curvature not less than 1 in the sense of Alexandrov), and $\left(M_{i}, d_{i}, p_{0}\right)$ are smooth with sec $\geq 0$. This is the situation examined in the introduction. It is well known that the space of directions $\Sigma_{0}(X)$ of $\left(X, d_{X}\right)$ at 0 is $\left(V, d_{V}\right)$ : see Theorem 10.9.3 (here we have used that the tangent cone of $X$ at 0 is equal to $X$, since $X$ is a cone). Now Theorem 5.1 of [22] says that $\Sigma_{0}(X)$ is homeomorphic to $\Sigma_{p_{0}} M_{i}$ (for $i$ big enough) which is isometric to the standard sphere $S^{n-1}$ since the $\left(M_{i}, g_{i}\right)$ are smooth manifolds. That is $V$ is homeomorphic to $S^{n-1}$.

\section{REFERENCES}

[1] S. Brendle, A generalization of hamilton's differential harnack inequality for the ricci flow, J. Differential Geom. 82 (2009), 207-227.

[2] D. Burago, Y. Burago, and S. Ivanov, A course in metric geometry, Graduate Studies in Mathematics, vol. 33, American Mathematical Society, Providence, RI, 2001.

[3] E. Cabezas-Rivas and B. Wilking, How to produce a ricci flow via cheeger-gromoll exhaustion, arXiv:1107.0606 3 .

[4] H-D. Cao, Limits of solutions to the Kähler-Ricci flow, J. Differential Geom. 45 (1997), no. 2, $257-272$.

[5] H-D. Cao, On dimension reduction in the Kähler-Ricci flow, Comm. Anal. Geom. 12 (2004), no. 1-2, 305-320.

[6] H-D. Cao, Recent progress on Ricci solitons, 2009, arXiv:0908.2006

[7] J. Carrilo and L. Ni, Sharp logarithmic Sobolev inequalities on gradient solitons and applications, Comm. Ana. Geom. 17 (2010), no. 4, 1-33. 
[8] A. Chau and L-F. Tam, On the simply connectedness of non-negatively curved Kähler manifolds and applications, arXiv:0806.2457 $\mathrm{J}$.

[9] J. Cheeger and T.H. Colding, On the structure of spaces with Ricci curvature bounded below. I, J. Differential Geom. 46 (1997), no. 3, 406-480.

[10] B-L. Chen and X-P. Zhu, Complete Riemannian manifolds with pointwise pinched curvature, Invent. Math. 140 (2000), no. 2, 423-452.

[11] B. Chow and R.S. Hamilton, Constrained and linear Harnack inequalities for parabolic equations, Invent. Math. 129 (1997), no. 2, 213-238.

[12] B. Chow, P. Lu, and L. Ni, Hamilton's Ricci flow, Graduate Studies in Mathematics, vol. 77, American Mathematical Society, Providence, RI, 2006.

[13] G. de Rham, Sur la reductibilité d'un espace de Riemann, Comment. Math. Helv. 26 (1952), 328-344.

[14] K. Ecker and G. Huisken, Interior estimates for hypersurfaces moving by mean curvature, Invent. Math. 105 (1991), no. 3, 547-569.

[15] M. Feldman, T. Ilmanen, and D. Knopf, Rotationally symmetric shrinking and expanding gradient Kähler-Ricci solitons, J. Differential Geom. 65 (2003), no. 2, 169-209.

[16] M. Feldman, T. Ilmanen, and L. Ni, Entropy and reduced distance for Ricci expanders, J. Geom. Anal. 15 (2005), no. 1, 49-62.

[17] L. Guijarro and V. Kapovitch, Restrictions on the geometry at infinity of nonnegatively curved manifolds, Duke Math. J. 78 (1995), no. 2, 257-276.

[18] R.S. Hamilton, Three-manifolds with positive Ricci curvature, J. Differential Geom. 17 (1982), no. 2, 255-306.

[19] R.S. Hamilton, Four-manifolds with positive curvature operator, J. Differential Geom. 24 (1986), no. 2, 153-179.

[20] R.S. Hamilton, A compactness property for solutions of the Ricci flow, Amer. J. Math. 117 (1995), no. 3, 545-572.

[21] R.S. Hamilton, The formation of singularities in the Ricci flow, Surveys in differential geometry, Vol. II (Cambridge, MA, 1993), Int. Press, Cambridge, MA, 1995, pp. 7-136.

[22] V. Kapovitch, Regularity of limits of noncollapsing sequences of manifolds, Geom. Funct. Anal. 12 (2002), no. 1, 121-137.

[23] O.A. Ladyženskaja, V.A. Solonnikov, and N.N. Ural'ceva, Linear and quasilinear equations of parabolic type, Translated from the Russian by S. Smith. Translations of Mathematical Monographs, Vol. 23, American Mathematical Society, Providence, R.I., 1967.

[24] M. Li, Ricci expanders and type III Ricci flow, arXiv:1008.0711

[25] L. Ni, A new matrix Li-Yau-Hamilton estimate for Kähler Ricci flow, arXiv:math/0502495 2.

[26] L. Ni and L-F. Tam, Kähler-Ricci flow and the Poincaré-Lelong equation, Comm. Anal. Geom. 12 (2004), no. 1-2, 111-141.

[27] G. Perelman, Alexandrov spaces with curvatures bounded from below II, unpublished typed manuscript.

[28] G. Perelman, The entropy formula for the Ricci flow and its geometric applications, arXiv:math/0211159

[29] W-X. Shi, Deforming the metric on complete Riemannian manifolds, J. Differential Geom. 30 (1989), no. 1, 223-301.

[30] M. Simon, Ricci flow of non-collapsed 3-manifolds whose Ricci-curvature is bounded from below, arXiv:0903.2142

[31] M. Simon, Ricci flow of almost non-negatively curved three manifolds, J. Reine Angew. Math. 630 (2009), 177-217.

[32] T. Yokota, Curvature integrals under the Ricci flow on surfaces, Geom. Dedicata 133 (2008), 169-179.

Felix Schulze: Freie Universität Berlin, Arnimallee 3, 14195 Berlin, Germany

E-mail address: Felix.Schulze@math.fu-berlin.de

Miles Simon: Universität Magdeburg, Universitätsplatz 2, 39106 Magdeburg, GerMANY

E-mail address: msimon@ovgu.de 